\title{
Block Copolymer Nanostructures Mapped by Far-Field Optics
}

\author{
Chaitanya K. Ullal, ${ }^{\dagger}$ Roman Schmidt, ${ }^{\dagger}$ Stefan W. Hell, ${ }^{*}$ and Alexander Egner \\ Department of NanoBiophotonics, Max Planck Institute for Biophysical Chemistry, Am \\ Fassberg 11, 37077 Göttingen, Germany
}

Received April 30, 2009

\begin{abstract}
We demonstrate stimulated emission depletion microscopy using opposing objective lenses to noninvasively reveal the nanoscale morphology of block copolymers in three dimensions with focused light. This is exemplified in a poly(styrene-block-2-vinylpyridine) model system in which contrast is achieved by specifically staining the vinylpyridine phase with a fluorescent dye. We image swelling induced mesopores and other convoluted structures within the bulk of samples, at scales that have so far required electron and scanning probe microscopes.
\end{abstract}

Block copolymers, which can self-assemble into a myriad of three-dimensional (3D) structures, constitute the key element of numerous nanotechnological applications. ${ }^{1-6} \mathrm{~A}$ block copolymer consists of two or more polymeric chains that are chemically different and covalently linked to each other. ${ }^{7}$ If the blocks are immiscible, enthalpy drives them to phase segregate, while entropic requirements promote mixing. The covalent connectivity of the blocks prevents complete separation, and under appropriate conditions, a balance of thermodynamic forces drives the segregation into a variety of ordered structures from a few to a few hundred nanometers in feature size. The equilibrium structure minimizes the unfavorable contact between the blocks without overstretching the chains, causing each block to occupy portions on opposite sides of an interface known as the intermaterial dividing surface (IMDS).

The geometry of the IMDS and thus the morphology depend on a variety of parameters including the number of blocks, the degree of polymerization, the extent to which the segments are incompatible, as well as on the volume fractions and molecular architecture of the components. Given the rich tapestry of potential structures that this palette of parameters leads to, tools that allow for the unambiguous and fast mapping of the 3D-morphology assume critical importance. While scattering based techniques allow for definitive morphological identification, including in situ measurements, they do not provide truly local structural information. ${ }^{8,9}$ In real space, this imaging has typically been performed with either electron or scanning probe microscopes. However, these techniques can only yield a 2D projection of thin sections or the morphology of surfaces. Variations of these methods, such as the reconstruction of

* Corresponding author, shell@gwdg.de.

$\uparrow$ C.K.U. and R.S.: equal contribution, random order.
3D structure from data obtained by undertaking by a tilt sequence of images in an electron microscope ${ }^{10}$ or by sequentially etching and imaging with a scanning probe microscope ${ }^{11}$ are limited by being restricted to sections thinner than $100 \mathrm{~nm}$ and being unable to image nondestructively, respectively. Recent efforts requiring the in situ determination of complete 3D structure have thus concentrated on the use of confocal microscopy. ${ }^{12,13}$ Since the resolution of this far-field optical method is limited by diffraction to $>180 \mathrm{~nm}$ in the focal plane and $>450 \mathrm{~nm}$ along the optic axis, one is restricted to examining polymers of ultrahigh molecular weight.

Recent advances in optical microscopy have shown that the diffraction resolution limit in fluorescence microscopy can be overcome by switching the fluorescence of the labels in use. ${ }^{14}$ For example, stimulated emission depletion microscopy (STED) produces fluorescence focal spots of subdiffraction dimensions by transiently switching off the fluorescence throughout the focal region except in a tiny area at the center of the excitation spot. To this end, the beam for fluorescence excitation is overlapped with the STED pattern, a red-shifted beam of light with an intensity zero, preventing fluorescence everywhere except at its very center. ${ }^{15,16}$ The resulting subdiffraction-sized fluorescence spot represents the effective point-spread-function (PSF) of the microscope. The more recent single molecule based concepts assemble an image from the coordinates of single fluorescence molecules that are stochastically switched in space. ${ }^{17-20}$ While both schemes can achieve 3D subdiffraction resolution, ${ }^{21-24}$ here we have opted for isoSTED ${ }^{24}$ microscopy, a powerful combination of STED and 4Pi microscopy using opposing objective lenses. ${ }^{25,24}$ Whereas in many variants of STED microscopy the fluorescence spot is compressed only axially $^{25}(z)$ or laterally ${ }^{26}(x y)$, here the STED beam is 


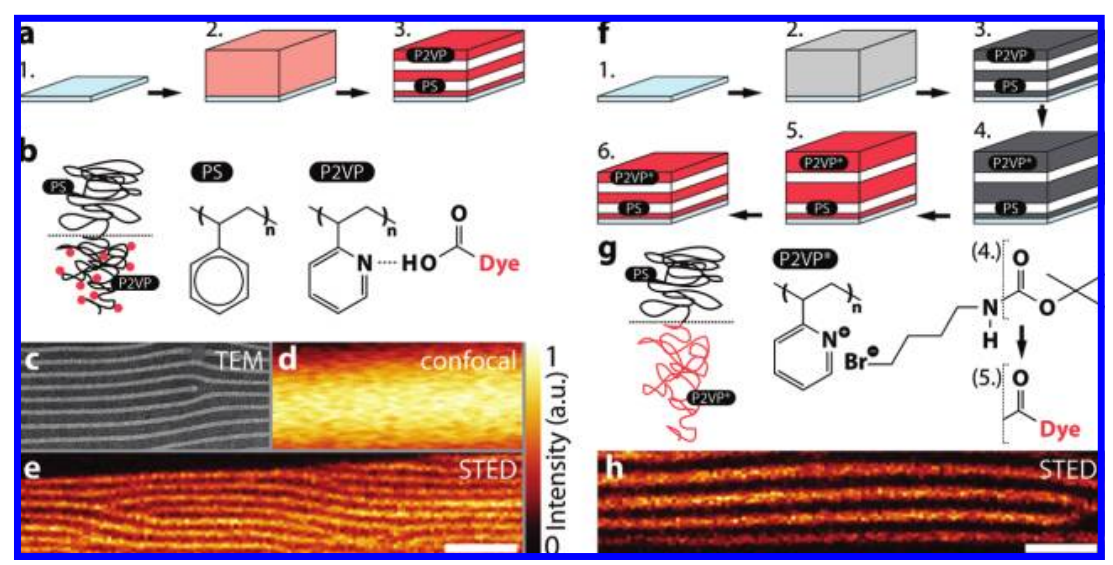

Figure 1. Sample processing and staining techniques. (a) A polymer solution containing dye is spun coat onto surface functionalized glass (1) to yield a uniform thin film (2). Phase separation is achieved by solvent annealing (3). (b) Confinement of the fluorescent marker to the $\mathrm{P} 2$ VP domain is achieved by stabilizing the solubility of the dye via a hydrogen bond. (c) Transmission electron micrograph of a solventannealed thin film showing the lamellar structure. (d) The confocal image of the block copolymer is essentially featureless while (e) the STED image clearly reveals the underlying morphology. (f) Polymer thin film processing and subsequent staining via chemical attachment. The film is spun coat (2), solvent annealed (3), quarternized (4), tagged with the dye (5), and dried. (g) The P2VP phase is connected to a bifunctional linker subsequent to phase separation by means of a quaternization reaction. Staining is achieved by reacting the deprotected amine at the end of the linker with an NHS ester of the fluorescent dye. (h) isoSTED image of the lamellar nanostructure. Scale bars, 500 nm.

prepared to form a hollow sphere thereby compressing the focal spot from all directions toward smaller dimensions. IsoSTED has three major advantages over its single molecule microscopy counterparts when imaging block copolymers in 3D: (i) it simultaneously collects the fluorescence from many fluorophores in the same focal spot, thus providing good contrast within a few seconds of recording, (ii) the focal spot can be scanned to any part in the sample, and (iii) the size and shape of the spot can be tuned in all three dimensions and thus adapted to the morphology investigated.

Fluorescence nanoscopy of block copolymers requires the generation of fluorescence contrast between the various phases. This fluorescence contrast can be achieved by exploiting spectral disparities of the dye in the different microdomain environments or by restricting fluorophores to definite phases. The latter strategy includes the incorporation of the dye into the polymer chain of one of the blocks during synthesis, the utilization of the preferential solubility of the dye in a particular phase, or the subsequent staining of the phases through physical or chemical bonds. In this work we utilize two out of these multiple staining options.

First, we labeled the phases by preferential dye solubility. As a model block copolymer system we chose an amorphous poly(styrene-block-2-vinylpyridine) system (PS-P2VP) which afforded well-defined lamellae in the bulk state as shown in Figure 1c. Solvent annealing ensured phase separation and was performed by placing spun coat films of the polymer doped with a dye, ATTO-647N $(\mathrm{a} 647 \mathrm{~N})$ in a vapor of pure chloroform at a fixed pressure ( $p=90$ Torr) and temperature $\left(T=23{ }^{\circ} \mathrm{C}\right)$, for $36 \mathrm{~h}$. Preferential solubility of the a647N in the P2VP phase was achieved by means of a stabilizing hydrogen bond between the fluorophore and the nitrogen atom in the pyridine group. ${ }^{27}$ To this end, a derivative dye bearing a hydroxyl group in the form of a carboxylic acid was used. A cartoon describing the processing is shown in parts $\mathrm{a}$ and $\mathrm{b}$ of Figure 1. A direct comparison of $x z$ cross sectional confocal and STED images from the same part of the block copolymer sample is shown in parts $d$ and e of Figure 1. While the confocal image is featureless, its STED counterpart clearly reveals the in-plane oriented lamellae of the film. The repeat unit of the lamellae is in good agreement with that seen in the electron micrograph.

An alternative staining approach, in which the P2VP block was tagged subsequent to phase separation, was equally viable (Figure $1 \mathrm{f}-\mathrm{h}$ ). Tagging was accomplished by reacting the block with one end of a bifunctional linker, the other end of which is a protected amino group. The polymer was then stained via the reaction of the deprotected amino group with an NHS ester of the fluorescent dye.

Given the range of morphologies that block copolymers can adopt, an important aspect of the isoSTED microscope is the control afforded in varying the shape and size of the PSF. We were able to engineer the intensity distribution of the STED beam, thereby customizing the shape of the focal spot of the isoSTED nanoscope to access spherical, oblate, and prolate point spread functions. The ability to adapt the spot with consideration to the examined morphology allowed us to tune to the optimum balance between resolution, signalto-noise ratio, and imaging speed. In practice, we typically chose a spherical spot for preliminary imaging and adopted a spot suitable for the particular case. Oblate, prolate, and spherical spots can be employed while imaging aligned lamellae, aligned cylinders, and disordered or bicontinuous morphologies, respectively. For example, while imaging the parallel layers in parts e and h of Figure 1, an oblate PSF was used. All morphologies shown throughout this letter were recorded utilizing pixel dwell times in the 1 to $10 \mu$ s range.

For morphologies that did not possess a preferred orientation, we employed the spherical PSF. To exemplify this, we imaged two kinetically trapped morphologies. Figure 2c shows an $x z$ section of a PS-P2VP polymer with a P2VP weight fraction of 0.3 . At this weight fraction we 


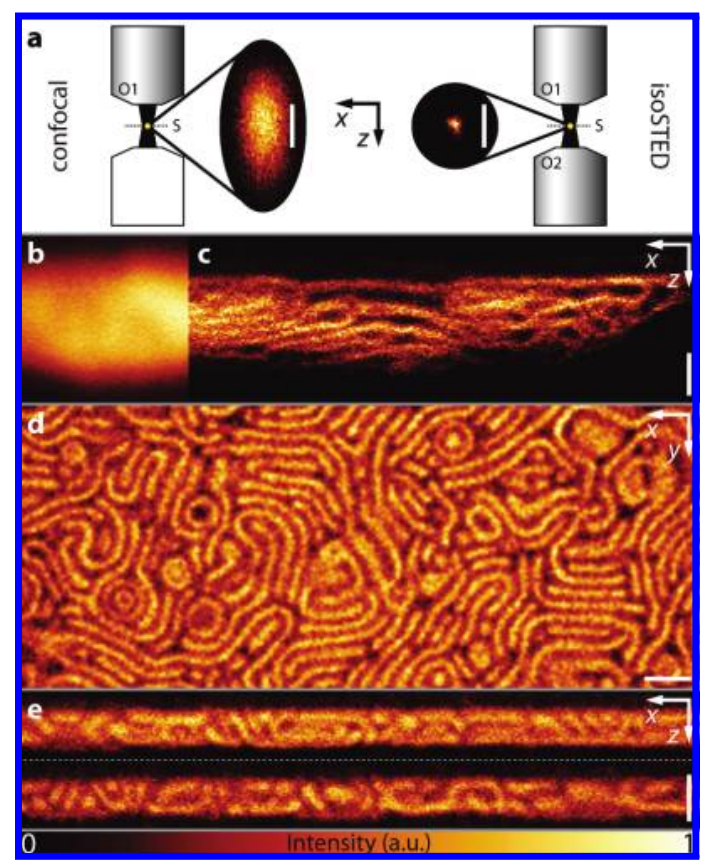

Figure 2. Imaging with an isotropic PSF. (a) $x z$ sections of typical confocal (left) and isoSTED (right) PSFs mapped by a fluorescent bead $\sim 40 \mathrm{~nm}$ in diameter. The isoSTED spot is created by the coherent use of the wavefronts of the two opposing objective lenses $\mathrm{O} 1$ and $\mathrm{O} 2$ which focus onto the same spot in the sample plane (S). (b) Confocal micrograph of a kinetically trapped structure seen in a PS-P2VP block copolymer is compared with (c) an isoSTED image from a contiguous region, recorded with a spherical spot $<50 \mathrm{~nm}$ in diameter. The weight fraction of the P2VP block is 0.3 . (d) $x y$ and (e) two $x z$ sections taken at different locations in a film of a PS-P2VP block copolymer, swollen with 30 wt \% PS homopolymer. The weight fraction of the PS-P2VP block copolymer is 0.5. Scale bars $250 \mathrm{~nm}$ (a) and 500 (c, d, and e).

expect a morphological transition from lamellae to cylinders. If probed with a confocal PSF, such as the image on the left of Figure 2a, the resultant image, Figure 2b, provides no information regarding the underlying structure. In stark contrast, a spherical PSF with diameter $<50 \mathrm{~nm}$, an example of which is shown on the right of Figure 2a, unravels the structure to reveal aligned lamellae and an interconnected structure on the left- and right-hand sides of the section, respectively. The second morphology probed using the sphere was obtained using PS-P2VP with a P2VP weight fraction of 0.5 that was swollen with homopolymer of PS so as to achieve a net weight fraction of 0.3 in P2VP. Images of sections taken perpendicular to the $z$ (Figure 2d) and $y$ (Figure 2e) axes, at different locations, show a kinetically trapped bicontinuous structure that exhibits both lamellar and worm-like features.

The unique strength of our method to unambiguously map the complete 3D structure within the bulk of a sample is further demonstrated by imaging a swelling-induced mesoporous morphology. Exposure of the block copolymer to a swelling agent that interacts primarily with one of the blocks significantly increases the volume of that phase. If the second phase is glassy, and therefore fixed, the rapid drying and concomitant collapse of the swollen phase can result in the formation of mesopores. ${ }^{28}$ Figure $3 \mathrm{~b}$ shows raw and dehazed

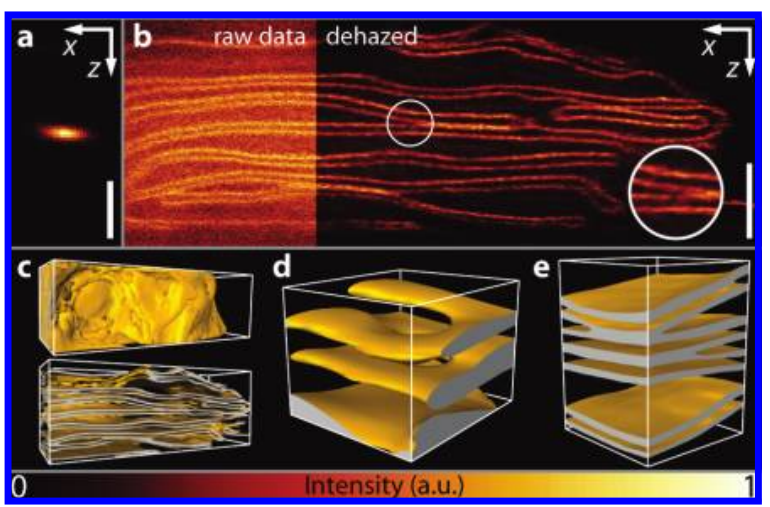

Figure 3. 3D reconstruction of swelling-induced mesoporous morphology recorded by STED microscopy. (a) Typical oblate PSF shape $(43 \times 120 \mathrm{~nm})$, mapped by a fluorescent bead $\sim 40 \mathrm{~nm}$ in diameter. (b) Raw (left) and dehazed (right) $x z$ section, taken from a 3D data stack recorded with an oblate PSF. As seen in the highlighted and $2 \times$ enlarged region, the unzipped layers lining the pores are on average over half the size of the intact ones. (c) Perspective views of the corresponding data stack, which was binarized to aid in the visualization. (d) A helicoidal screw dislocation neighboring a pore. (e) Selected area showing "unzipping" of the P2VP domains, forming pores. Scale bars, $250 \mathrm{~nm}$ (a) and $1 \mu \mathrm{m}$ (b). Length of short edge was $2.5 \mu \mathrm{m}$ (c), $0.6 \mu \mathrm{m}$ (d), and $1.0 \mu \mathrm{m}$ (e).

views of a $x z$ section taken in a mesoporous sample prepared using water as the selective swelling solvent. The section reveals unzipped quaternized P2VP lamellae lining large dark pores created by the water, with intact alternating layers of PS and P2VP between them. The unzipped lamellae are seen to be on average over half the thickness of the intact P2VP domains.

To aid in the visualization of the complete 3D structure, the data set was subsequently binarized, a rendering of which is shown in Figure 3c. Panels e and d of Figure 3 are blow ups of selected areas showing the morphology of a typical mesopore and a helicoidal screw dislocation, respectively. Such dislocations have been suggested as the path for rapid solvent transport in reversibly swellable photonic crystal gels. ${ }^{29}$

These fluorescence nanoscopy images of block copolymer nanostructures are notable for the possibilities that they create. The noninvasive access to morphological information within the bulk of the sample, with microdomain specificity, coupled with the demonstrated ability of STED microscopy to achieve both multiple colors, ${ }^{30}$ as well as fast scanning ${ }^{31}$ will enable numerous fundamental studies. Examples of structural studies include the unambiguous mapping of the architecture of multicomponent block polymers, the imaging of the structural nature of defects, and the effect of 3D confinement on block copolymer morphology. The dynamic studies should include elucidation of the mechanisms and dynamics behind the process of ordering in self-assembly under various driving forces such as electric fields and graphoepitaxy. We therefore anticipate that far-field optical nanoscopes, and STED microscopes in particular, will join electron microscopes and X-ray scattering instruments as key tools for polymer scientists. 
Acknowledgment. This work was supported by a grant from the Alexander von Humboldt Foundation to C.K.U. and by a grant from the Deutsche Forschungsgemeinschaft (SFB 755) to A.E. and S.W.H. We gratefully acknowledge helpful discussions with Lars Karstrup (MPIbpc) and Edwin Chan (NIST) related to the attachment of fluorophores and polymer processing, respectively. This work benefitted from preliminary collaborative work with Douglas Adamson (University of Connecticut). We thank Donald Ouw (MPIbpc) for excellent technical assistance and Dietmar Riedel (MPIbpc) for performing the transmission electron microscopy.

Supporting Information Available: Details of materials used and experimental procedures. This material is available free of charge via the Internet at http://pubs.acs.org

\section{References}

(1) Park, C.; Yoon, J.; Thomas, E. L. Polvmer 2003, 44 (22), 6725-6760.

(2) Park, M.; Harrison, C.; Chaikin, P. M.; Register, R. A.; Adamson, D. H. Science 1997, 276 (5317), 1401-1404.

(3) Stoykovich, M. P.; Muller, M.; Kim, S. O.; Solak, H. H.; Edwards, E. W.; de Pablo, J. J.; Nealey, P. F. Science 2005, 308 (5727), 14426.

(4) Black, C. T.; Ruiz, R.; Breyta, G.; Cheng, J. Y.; Colburn, M. E.; Guarini, K. W.; Kim, H.-C.; Zhang, Y. IBM J. Res. Dev. 2007, 51 (5), 605-633.

(5) Fink, Y.; Urbas, A. M.; Bawendi, M. G.; Joannopoulos, J. D.; Thomas, E. L. J. Lightwave Technol. 1999, 17 (11), 1963-1969.

(6) Vriezema, D. M.; Aragones, M. C.; Elemans, J. A. A. W.; Cornelissen, J. J. L. M.; Rowan, A. E.; Nolte, R. J. M. Chem. Rev. 2005, 105 (4), $1445-1490$

(7) Bates, F. S.; Fredrickson, G. H. Phvs. Todav 1999, 52 (2), 32-38.

(8) Smilgies, D. M.; Busch, P.; Papadakis, C. M.; Posselt, D. Synchrotron Radiation News 2002, 15 (5), 35-42.

(9) Xu, T.; Goldbach, J. T.; Misner, M. J.; Kim, S.; Gibaud, A.; Gang, O.; Ocko, B.; Guarini, K. W.; Black, C. T.; Hawker, C. J.; Russell, T. P. Macromolecules 2004, 37 (8), 2972-2977.

(10) Spontak, R. J.; Williams, M. C.; Agard, D. A. Polvmer 1988, 29, 387.
(11) Magerle, R. Phvs. Rev. Lett. 2000, 85 (13), 2749-2752.

(12) Yoon, J.; Lee, W.; Thomas, E. L. Adv. Mater. 2006, 18 (20), 26912694.

(13) Lee, W.; Yoon, J.; Lee, H.; Thomas, E. L. Macromolecules 2007, 40 (17), 6021-6024.

(14) Hell, S. W. Science 2007, 316 (5828), 1153-1158.

(15) Hell, S. W.; Wichmann, J. Opt. Lett. 1994, 19 (11), 780-782.

(16) Klar, T. A.; Jakobs, S.; Dyba, M.; Egner, A.; Hell, S. W. Proc. Natl. Acad. Sci. U.S.A 2000, 97, 8206-8210.

(17) Rust, M. J.; Bates, M.; Zhuang, X. Nat. Methods 2006, 3, 793-796.

(18) Betzig, E.; Patterson, G. H.; Sougrat, R.; Lindwasser, O. W.; Olenych, S.; Bonifacino, J. S.; Davidson, M. W.; Lippincott-Schwartz, J.; Hess, H. F. Science 2006, 313 (5793), 1642-1645.

(19) Hess, S. T.; Girirajan, T. P. K.; Mason, M. D. Biophys. J. 2006, 91 (11), 4258-4272.

(20) Egner, A.; Geisler, C.; von Middendorff, C.; Bock, H.; Wenzel, D.; Medda, R.; Andresen, M.; Stiel, A.-C.; Jakobs, S.; Eggeling, C.; Schönle, A.; Hell, S. W. Biophvs. J. 2007, 93, 3285-3290.

(21) Harke, B.; Ullal, C. K.; Keller, J.; Hell, S. W. Nano Lett. 2008, 8 (5), 1309-1313.

(22) Huang, B.; Wang, W.; Bates, M.; Zhuang, X. Science 2008, 319, 810813.

(23) Juette, M. F.; Gould, T. J.; Lessard, M. D.; Mlodzianoski, M. J.; Nagpure, B. S.; Bennett, B. T.; Hess, S. T.; Bewersdorf, J. Nat. Methods 2008, 5 (6), 527-529.

(24) Schmidt, R.; Wurm, C. A.; Jakobs, S.; Engelhardt, J.; Egner, A.; Hell, S. W. Nat. Methods 2008, 5 (6), 539-544.

(25) Dyba, M.; Hell, S. W. Phvs. Rev. Lett. 2002, 88, 163901.

(26) Willig, K.; Keller, J.; Bossi, M.; Hell, S. W. New J. Phvs. 2006, 8, 106.

(27) Nakashima, K.; Winnik, M. A.; Dai, K. H.; Kramer, E. J.; Washiyama, J. Macromolecules 1992, 25 (25), 6866-6870.

(28) Wang, Y.; Gösele, U.; Steinhart, M. Nano Lett. 2008, 8 (10), 35483553.

(29) Kang, Y.; Walish, J. J.; Gorishnyy, T.; Thomas, E. L. Nat. Mater. 2007, 6 (12), 957-960.

(30) Donnert, G.; Keller, J.; Wurm, C. A.; Rizzoli, S. O.; Westphal, V.; Schönle, A.; Jahn, R.; Jakobs, S.; Eggeling, C.; Hell, S. W. Biophvs. J. 2007, 92 (8), L67-L69.

(31) Westphal, V.; Rizzoli, S. O.; Lauterbach, M. A.; Kamin, D.; Jahn, R.; Hell, S. W. Science 2008, 320 (5873), 246-249.

NL901378E 